НИКОЛЬСКИЙ Николай Игоревич - сотрудник ООО «ТЕХНОС-К», исполнитель проекта Российского фонда фундаментальных исследований (РФФИ) (119334, Россия, г. Москва, Ленинский np-кm, 32A; nickola_n@mail.ru)

РУБВАЛЬТЕР Дмитрий Александрович - доктор экономических наук, профессор; руководитель $и$ координатор проекта Российского фонда фундаментальных исследований (РФФИ) (119334, Россия, г. Москва, Ленинский пр-кт, 32A; dmitry.rubvalter@yandex.ru)

РУДЕНСКИЙ Олег Владимирович - кандидат экономических наук, исполнитель проекта Российского фонда фундаментальных исследований (РФФИ) (119334, Россия, г. Москва, Ленинский np-кm, 32A; trud55@mail.ru)

\title{
РАЗРАБОТКА ИНФОРМАЦИОННОЙ И ПРОГРАММНО-АНАЛИТИЧЕСКОЙ СИСТЕМЫ «ЦИФРОВЫЕ СТРАТЕГИИ» РОССИЙСКОЙ ФЕДЕРАЦИИ (І ЭТАП)
}

\begin{abstract}
Аннотация. Авторы в развитие предыдущего проекта РФФИ (см. «Власть» № 3 за 2019 г.) подготовили данную статью, охватывающую цели, задачи и практическую реализацию нового проекта РФФИ «Междисциплинарное исследование процессов цифровизации, компьютеризации, измерения и оценки роли науки при разработке и реализации политических решений». В статье представлены основные идеи, принципы и инструментарий создания информационной и программно-аналитической системы «Цифровые стратегии» России (СЦС). Предполагается ее временная эксплуатация в Интернете и подсоединение к этому процессу заинтересованных министерств. В этом контексте, как считают авторы, целесообразно будет поставить вопрос о подсоединении СЦС к государственной автоматизированной системе «Управление» в разделе федеральной информационной системы стратегического планирования (ФИС СП). В статье авторы показывают структуру и содержание функциональной, информационной и программно-аналитической платформы СЦС, а также тематику проектов и вопросников. В качестве основной нормативной базы построения СЦС авторы принимают федеральный закон от 28 июня 2014 г. № 72-Ф3 “О стратегическом планировании в Российской Федерации». Помимо федерального закона, в своей работе авторы опираются на соответствующие указы Президента РФ и Правительства РФ, относящиеся к организации проведения обсуждений стратегий, прогнозов и их последующего мониторинга. В статье авторы показывают этапы и основные направления автоматизации этого процесса и подготовки программно-компьютерного обеспечения. Данная работа будет проводиться в течение трех лет - с 2020 по 2022 г.
\end{abstract}

Ключевые слова: стратегическое планирование, система цифровых стратегий (СЦС), функциональное и программное обеспечение стратегического планирования, автоматизация разработки стратегий

$\mathrm{P}$ азработка политико-стратегических и нормативно-правовых документов - масштабный, многофункциональный, междисциплинарный, довольно сложный процесс, представляющий собой композитную, системную структуру. Она формируется не только в результате интеграции множества мнений, концепций и идей ученых и профессиональных специалистов, но и на основе общественных суждений, мнений, оценок представителей бизнеса, политических и профсоюзных деятелей, администрации министерств, ответственных за выполнение данной работы. Еще шесть лет назад был принят федеральный закон от 28 июня 2014 г. №72-Ф3 «О стратегическом планировании в Российской Федерации», одна из статей которого определяет структурный порядок построения стратегий, а также необходимость общественного обсуждения стратегических документов с использованием Интернета. В этом кон- 
тексте в соответствии с постановлением Правительства РФ $\Phi^{1}$ создан сайт государственной автоматизированной системы «Управление» 2 .

Важный новый элемент системы «Управление» - это стратегическое планирование (стратпланирование), отвечающее целям и задачам упомянутого федерального закона № 72-Ф3, касающимся организации и проведения общественного обсуждения процесса разработки и реализации политико-стратегических документов. Такая работа уже ведется Минэкономразвития РФ. Так, с 2019 г. осуществляются мероприятия по информационному обеспечению стратпланирования, входящему в проект «Цифровое стратпланирование». Создается система стратегического планирования ФИС СП. В этом контексте мы предлагаем возможное системное дополнение к ФИС СП под названием «Цифровые стратегии», которое могло бы встраиваться в автоматизированную систему «Управление» или существовать отдельно на специальном сайте Интернета для проведения комплексных проблемных оценок и мониторинга при разработке стратегий.

\section{1. Общие принципы построения системы «Цифровые стратегии»}

Исходя из сложности проблемы стратегического планирования, в рамках РФФИ осушествляется научное исследование по теме «Междисциплинарное исследование процессов цифровизации, компьютеризации и измерения оценки роли науки при разработке и реализации политических решений» (проект № 20-010-00179а) на базовой основе ЦЭМИ РАН. На основе этого исследования предполагается разработать информационную и программноаналитическую систему, в рамках которой могли бы осуществляться цифровые, тексто-смысловые и композитные оценки функциональных компонентов и показателей проблем политико-стратегических и нормативно-правовых документов. Эта система названа нами Системой Цифровых Стратегий (СЦС). Имеется в виду ее целевая функция, направленная на получение цифровых оценок стратегического планирования. Инструментарий этих оценок будет базироваться на различных типах вопросников. Ниже представлен титульный лист СЦС, имеющий определенное идейное и проблемно-смысловое значение в контексте статпланирования.

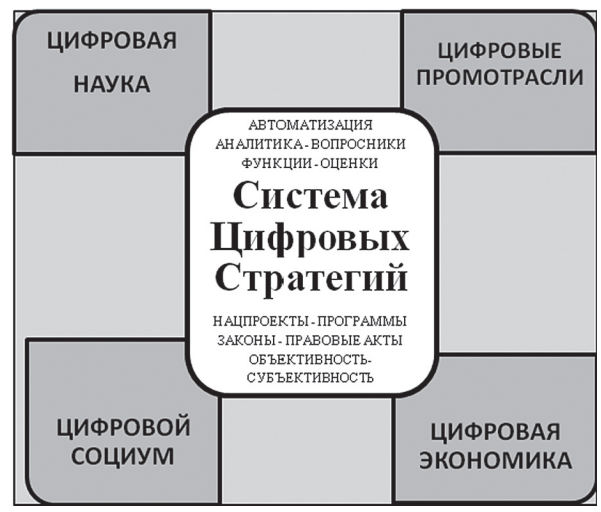

Рисунок 1. Титульный лист Системы Цифровых Стратегий

1 Постановление Правительства РФ от 25.12.2009 № 1088 « государственной автоматизированной системе «Управление» (с изм. от 18.11.2019 № 1488). Доступ: https:// base.garant.ru/197043/ (проверено 30.10.2020).

2 Государственная автоматизированная система «Управление». Доступ: http://gasu.gov.ru/ stratpassport (проверено 30.10.2020). 
СЦС включает четыре блока стратпланирования, охватывающих сферу цифровых социально-экономических, научно-технологических и промышленноотраслевых проблем, относящихся к стратегиям страны, в частности к проекту «Цифровая экономика РФ». Именно эта структура определяет тематику и содержание вопросников - основополагающего инструментария СЦС для определения функционально-проблемных и тексто-смысловых оценок разрабатываемых и мониторинговых стратегий.

2. Функциональная и программно-аналитическая платформа Системы Цифровых Стратегий

СЦС - это размещаемый в Интернете композитный, междисциплинарный, научный программно-аналитический комплекс, сочетающий в себе интеллектуально-когнитивную систему научно-общественных дискуссий, цифровизацию и автоматизацию оценок многофункциональных компонентов и проблем социума, экономики, науки и отраслей промышленности.

С функциональной точки зрения СЦС разделяется на три этапа научнообщественных обсуждений (с 2020 по 2022 г.), которые отличаются друг от друга как набором проектов с различной проблемной тематикой, обозначаемых поэтапно вариантами исследования В-1, B-2 и В-3, так и применяемым на этих этапах различным инструментарием (различные типы вопросников и системы оценок), а также соответствующими проектами (см. рис. 2). Тематика и содержание четырех проектов, представленных в рамках варианта B-1, являются лишь первичной структурой. Предусматривается возможность введения дополнительных проектов по функционально-проблемным темам со стороны официальных представителей министерств и ведомств (администраторов СЦС), ответственных за разработку тех или иных стратегий и осуществляющих подготовку и проведение научно-общественного опроса. На втором и третьем этапе варианты проектов В-2 и В-3 будут детально рассмотрены в 2021 и 2022 гг.

Важный элемент СЦС - это вопросники, которые являются основой для получения оценок различных элементов разрабатываемых стратегий и прогнозов. Исходя из наличия трех этапов и трех типов вариантов, применяются следующие три типа вопросников.

Функционально-проблемные вопросники балльных оценок. Структурно они представляют собой (вариант В-1) систему для получения балльных оценок стратегических проблем, объединяемых в функциональные блоки и показатели, отражающие комплексное проблемное содержание проектов варианта В-1 (см. рис. 2).

Вопросники тексто-смысловых оценок второго этапа опроса (вариант В-2) (см. рис. 2) предназначены для получения текстовых оценок для отбора и закрепления их в целях, задачах и приоритетах социально-экономических, научнотехнологических и отраслевых стратегий и прогнозов, на основе закона № 72-Ф3.

Мониторинговые функционально-проблемные и тексто-смысловые вопросники третьего этапа опроса (вариант В-3) предназначены для получения оценок мониторинга принятых к реализации соответствующих социально-экономических, научно-технологических и отраслевых стратегий и прогнозов.

В таблице 1 в качестве примера дано построение одного из элементов функционально-проблемного вопросника к проекту В1-1 «Цифровая оценка функциональных компонентов и показателей политик (стратегий) социального прогресса, качества жизни и человеческого развития РФ» (классификация оценок по 100-балльной системе). 


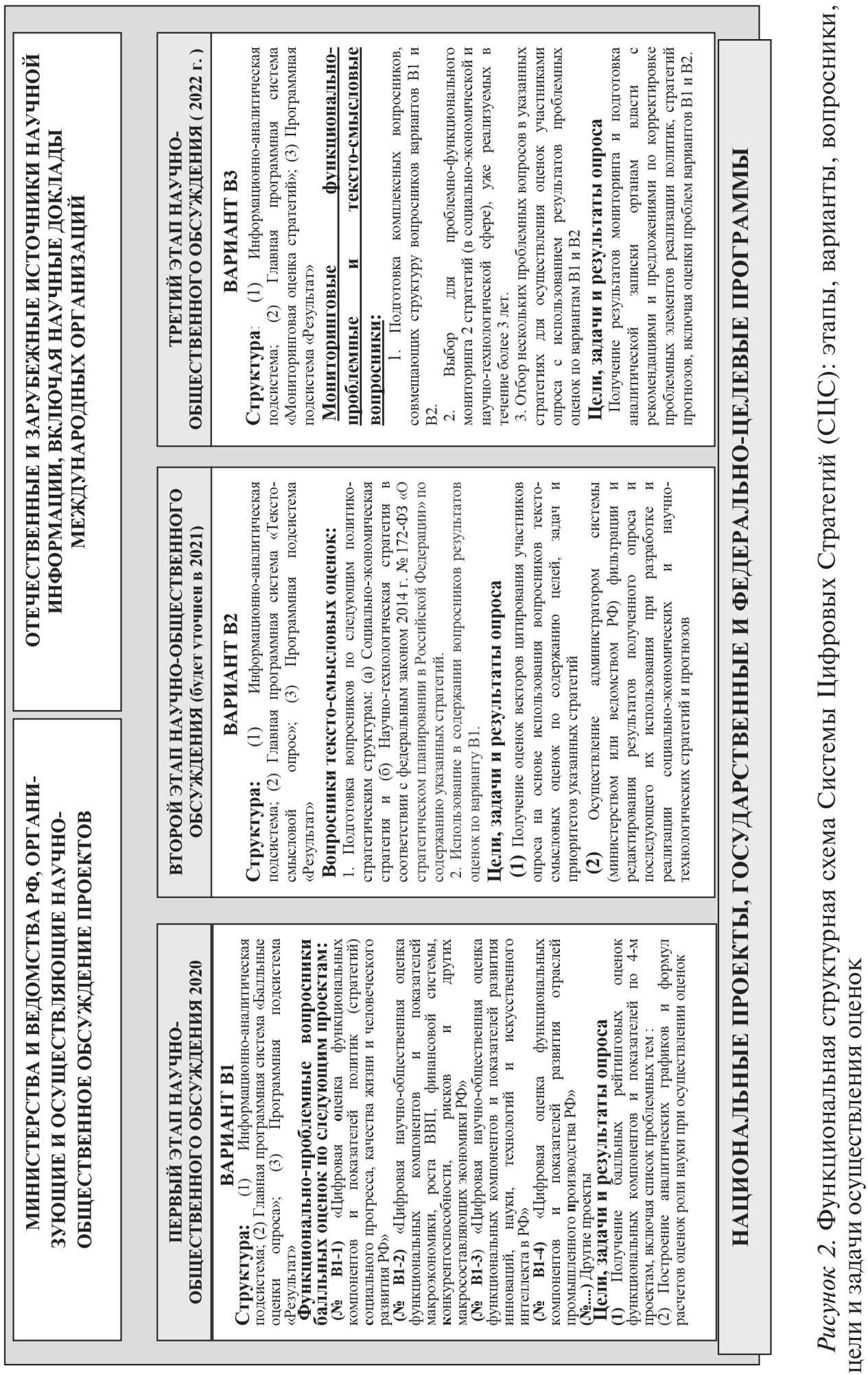


Таблица 1

Демонстрационный пример элемента функционально-проблемного вопросника по проекту В1-1

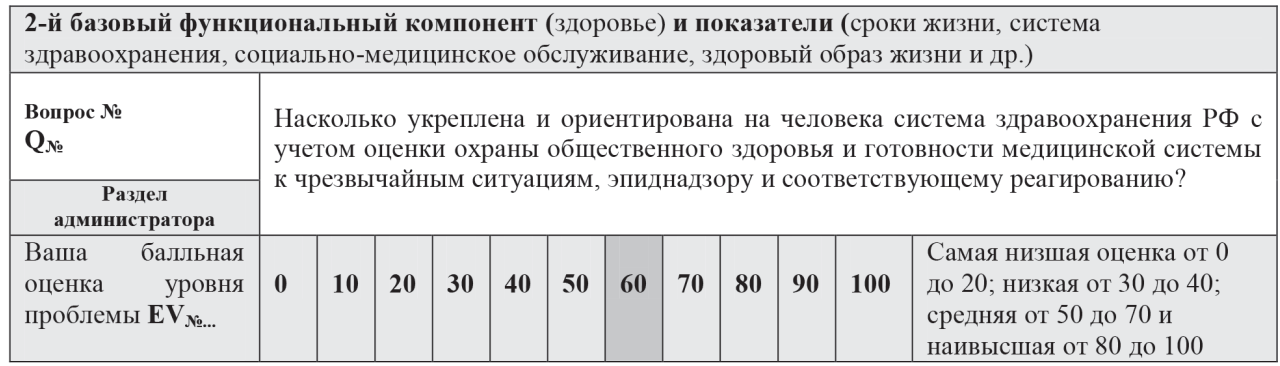

Вопросники СЦС выстраиваются структурно на основе базовых функциональных компонентов и показателей. Кроме того, вопросники строятся по принципу выделения базовых функциональных компонентов выбранной для исследования оценочной проблемы и отдельных показателей этой проблемы. Так, вопросник проекта В1-1, охватывающего социальные проблемы, имеет 5 базовых функциональных компонентов и значительное число показателей (вопросов). К базовым функциональным компонентам относятся питание, здоровье, жилище, социум и образование. Эти компоненты включают 84 вопроса. Вопросник проекта В1-2 по макроэкономике имеет более 100 вопросов.

Из опыта зарубежных стран и Всемирного экономического форума в Давосе вопросники могут включать до 100 вопросов и более и иметь соответствующую систему построения балльных оценок. Также используется довольно много глобальных индексов. Отношение к ним различное. Одни считают, что найден интегральный, композитный инструмент оценок функциональных показателей экономики, социума, науки, промышленности и т.д., а другие отрицают это.

Для каждого функционально-проблемного вопросника готовятся информационно-аналитические и библиометрические справки, показывающие основные принципы построения и содержания вопросников. Справки готовятся с учетом политико-стратегических документов, научных статей, докладов и монографий из российских и зарубежных источников, таких как $\mathrm{OOH}, \mathrm{EC}$, ОЭСР и др. Эти справки предназначены для участников опроса и учитывают различный уровень их подготовки, знаний и разные области интересов.

Общая информационная и программно-аналитическая платформа СЦС состоит из трех главных базовых систем и нескольких подсистем (см. рис. 3).

К главным базовым программным системам относятся: «Балльные оценки опроса» (I этап 2020 г., вариант В-1), «Тексто-смысловой опрос» (II этап, вариант В-2) и «Мониторинговая оценка стратегий» (III этап, вариант В-3). В каждую из базовых систем входят информационно-аналитические подсистемы и программные подсистемы «Результат».

В программно-аналитической системе СЦС после окончания научно-общественного опроса предусматривается автоматическое определение среднеарифметических значений оценок проектов, базовых функциональных компонентов и показателей вопросников, а также получение списка показателей с самыми низшими оценками, что дает возможность готовить соответствующие записки органам власти с рекомендациями по исправлению ситуации. 


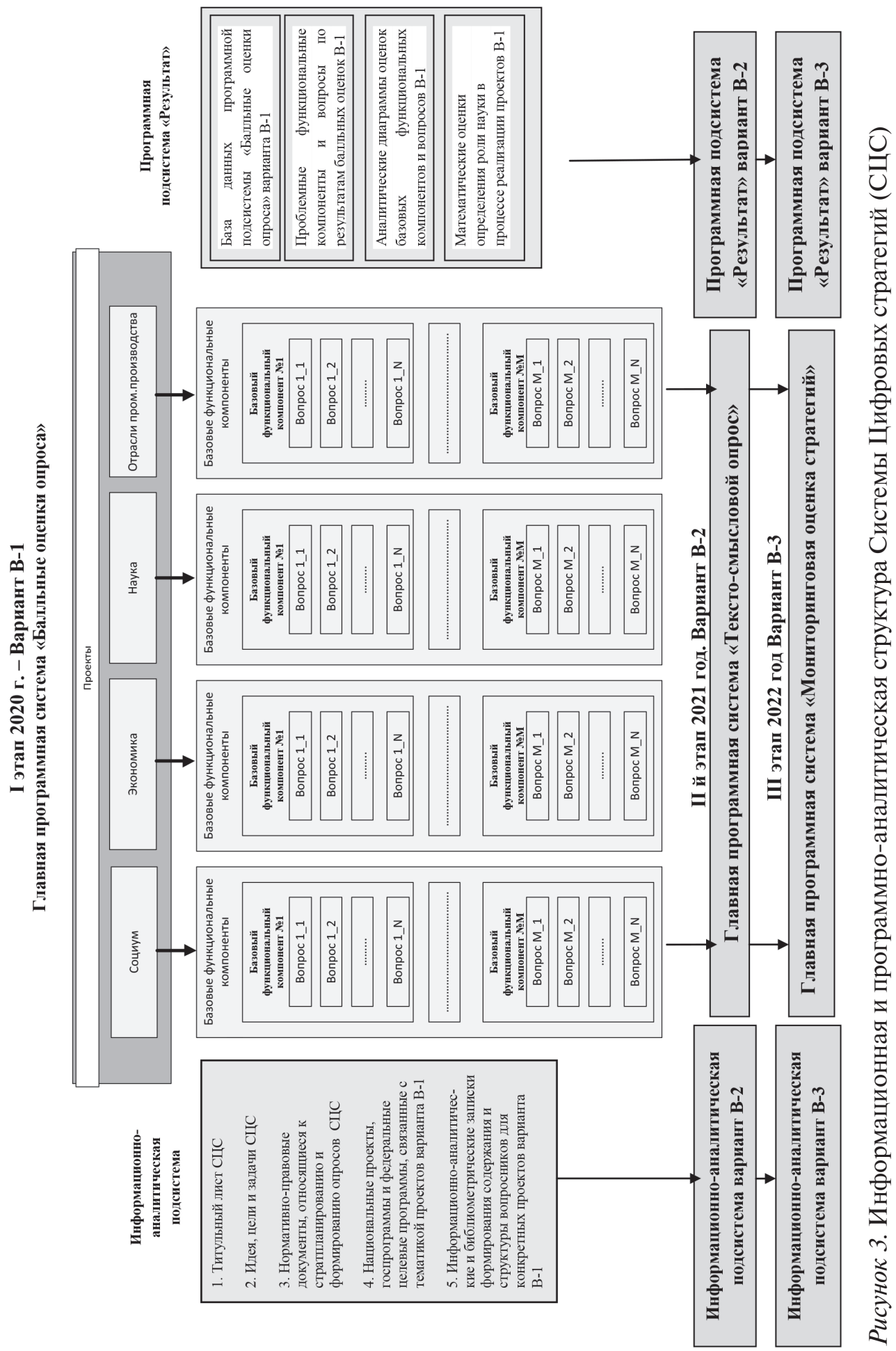


Кроме того, в системе СЦС предусматривается автоматическое построение диаграмм с цифровыми значениями функциональных компонентов и показателей, полученных в результате опроса, а также некоторые математические формулы для определения роли и эффективности науки при проведении научно-общественного опроса. Планируется, что система СЦС будет в предварительном порядке размещена в Интернете. Впоследствии предполагается поставить вопрос о возможном подсоединении СЦС к государственной автоматизированной системе «Управление» в раздел ФИС СП. По окончании работ будут подготовлены Методика и Инструкция по работе с информационной и программно-аналитической Системой Цифровых Стратегий.

Статья подготовлена при финансовой поддержке Российского фонда фундаментальных исследований (проект №20-010-00179a).

NIKOL'SKY Nikolai Igorevich, Employee of LLC «TECHNOS-K», Executor at the Russian Foundation for Basic Research(32A Leninsky Ave, Moscow, Russia, 119334; nickola_n@mail.ru)

RUBVAlTER Dmitriy Aleksandrovich, Dr.Sci. (Econ.), Professor; Project Head and Coordinator at the Russian Foundation for Basic Research (32A Leninsky Ave, Moscow, Russia, 119334; dmitry.rubvalter@yandex.ru)

RUDENSKY Oleg Vladimirovich, Cand.Sci. (Econ.), Senior Project Executor at the Russian Foundation for Basic Research (32A Leninsky Ave, Moscow, Russia, 119334 trud55@mail.ru)

\title{
THE DEVELOPMENT OF THE INFORMATION AND SOFTWARE-ANALYTICAL SYSTEM «DIGITAL STRATEGIES» OF THE RUSSIAN FEDERATION (THE 1 ST STAGE)
}

\begin{abstract}
The authors, in development of the previous Russian Fund for Basic Research (RFBR) project prepared this article covering goals, objectives and practical implementation of the new RFBR project «Interdisciplinary study of digitalization, computerization, measurement and assessment of the role of science in the development and implementation of political decisions". The article presents the basic ideas, principles and tools for creating the information and software-analytical system «Digital Strategies» of Russia (SDS). It is assumed that it will be temporarily operated on the Internet on the base of the Central Economic and Mathematic Institute website. It is assumed that some interested ministries will be connected to this process. In this context, according to the authors, it would be advisable to raise the question of connecting the SDS to the state-owned automated control system "Governance» in the section of the federal strategic planning information system. The authors show the structure and content of the functional, informational and software-analytical platform of the SDS, as well as the topics of projects and questionnaires. The main regulatory framework for constructing the SDS is the Federal Law No. 72-FZ of June 28, 2014 "On Strategic Planning in the Russian Federation». The authors in their work also relied on relevant decrees of the President of the RF and the Government of the RF relating to the organization of discussions on strategies, forecasts and their subsequent monitoring. On this base, the authors show the stages and main directions of automation of this process and the preparation of the computer software. This work will be carried out for 3 years (20202022).
\end{abstract}

Keywords: strategic planning, digital strategy system (SDS), functional and software for strategic planning, automation of strategy development 